\title{
OPEN Honey bee sHSP are responsive to diverse proteostatic stresses and potentially promising biomarkers of honey bee stress
}

\author{
Samantha R. Shih, Dunay M. Bach, Nicole C. Rondeau, Jessica Sam, Natalie L. Lovinger, \\ Allison J. Lopatkin \& Jonathan W. Snow
}

The pollination services provided by the honey bee are critical in both natural and agricultural ecosystems. Honey bee colonies in the United States have suffered from an increased rate of die-off in recent years, stemming from a complex set of interacting stresses that remain poorly described. Defining specific common cellular processes and cellular stress responses impacted by multiple stressors represent a key step in understanding these synergies. Proteotoxic stresses negatively impact protein synthesis, folding, and degradation. Diverse proteotoxic stresses induce expression of genes encoding small heat shock proteins (SHSP) of the expanded lethal (2) essential for life (l(2) efl) gene family. In addition to upregulation by the Integrated Stress Response (ISR), the Heat Shock Response (HSR), and the Oxidative Stress Response (OSR), our data provide first evidence that sHSP genes are upregulated by the Unfolded Protein Response (UPR). As these genes appear to be part of a core stress response that could serve as a useful biomarker for cellular stress in honey bees, we designed and tested an RT-LAMP assay to detect increased $l(2)$ efl gene expression in response to heatstress. While this assay provides a powerful proof of principle, further work will be necessary to link changes in SHSP gene expression to colony-level outcomes, to adapt our preliminary assay into a Point of Care Testing (POCT) assay appropriate for use as a diagnostic tool for use in the field, and to couple assay results to management recommendations.
Abbreviations
HSR Heat shock response
ISR Integrated stress response
OSR Oxidative stress response
UPR Unfolded protein response
sHSP Small heat shock protein

Honey bee pollination services are critical for the health of both agricultural and ecological systems ${ }^{1}$. Honey bee colonies in the United States have been lost at an increased rate in since the mid-2000s. Stressors including nutritional deficiencies due to loss of appropriate habitat, toxicity from pesticides, changes to normal living conditions brought about through industrial beekeeping practices, a host of environmental changes due to climate change, and parasitism by arthropod pests and pathogenic microbes, likely work in concert to cause tissue pathology, disease and mortality ${ }^{2}$. While studies on the physiological stress responses in the honey bee have provided significant insight ${ }^{3}$, we are only beginning to understand honey bee cellular stress responses, especially at the molecular level. Attempts to more completely define common cellular processes and cell stress pathways have been undertaken to better understand how stresses might work together to cause honey bee disease.

Proteostasis, which is defined as the homeostasis of protein synthesis, folding, function, and degradation, represents one such process ${ }^{4}$. This process can be disrupted by a number of normal and pathologic conditions, ultimately leading to an accumulation of misfolded proteins in the cell ${ }^{4}$. The pathways of the proteostatic network maintain protein homeostasis in individual cells. These responses include the Unfolded Protein Response of the endoplasmic reticulum (UPR), which responds to proteostatic perturbation in this compartment ${ }^{5}$, the Integrated 
Stress Response (ISR), which responds to amino acid deprivation or ribosome dysfunction, and the Heat Shock Response (HSR), responding to disruption of proteostasis in the cytoplasm.

We recently used an unbiased approach to identify novel genes altered in expression during induction of these proteostatic network responses in the honey bee using transcriptome profiling (RNASeq). We used tunicamycin, which disrupts $\mathrm{N}$-glycosylation in the ER to induce the UPR $\mathrm{UP}^{6}$. We also used thermal stress which causes protein denaturation and found that this induces a tissue regeneration program ${ }^{7}$. Finally, we used halofuginone, which inhibits the prolyl-tRNA synthetase to induce ISR, and observed increased expression of ribosome biogenesis genes $^{8}$. We hypothesized that we could then utilize this information to better understand how these stresses interact to impact honey bee health and to identify candidate biomarkers of stress in honey bees.

\section{Materials and methods}

Honey bee tissue collection. Honey bees represent a typical mix of Apis mellifera subspecies found in North America. They were collected from the landing board of outbred colonies in New York, New York at different times during the months of April-October. Only visibly healthy bees were selected and all source colonies were routinely inspected for symptoms of common bacterial, fungal, and viral diseases of honey bees. All bees used in a given experiment were taken from the same colony. Following cold anesthesia, the following tissues were dissected for gene expression analysis: head tissue (predominantly brain, sensory organ tissue, and hypopharyngeal glands), midgut, thorax tissue (predominantly flight muscle), and abdominal wall tissue (predominantly fat body). Dissected tissues were immersed in RNAlater (Invitrogen, San Diego, CA) for storage prior to RNA extraction.

Temperature and chemical treatments. Honey bees collected as above were maintained in $177.4 \mathrm{~mL}$ (6 oz.) Square-bottomed Drosophila Stock Bottles (VWR) plugged foam tube plugs (Jaece Industries, North Tonawanda, NY) modified to allow for feeding via microcentrifuge tubes as previously described ${ }^{9}$. Caged bees were maintained in incubators at $35^{\circ} \mathrm{C}$ (except for during heat shock as described below). PseudoQueen strips (Contech, Victoria, British Columbia, Canada) were placed in the incubators as a source of Queen Mandibular Pheromone (QMP). Heat shocked bees were maintained for $4 \mathrm{~h}$ in cages at $45^{\circ} \mathrm{C}$. Bees were fed $33 \%$ sucrose via a modified $1.5 \mathrm{ml}$ microcentrifuge tube, with $0.01-2.5 \mathrm{mM}$ paraquat, $0.0001-0.001 \% \mathrm{NaArsenite}, 24 \mu \mathrm{M}$ tunicamycin, $200 \mu \mathrm{M}$ halofuginone, or $0.5 \mathrm{mg} / \mathrm{ml}$ cycloheximide for $24 \mathrm{~h}$. For controls, bees were fed sucrose solution alone for paraquat, $\mathrm{NaArsenite}$ or cycloheximide. As the solvent for tunicamycin and halofuginone is DMSO, equivalent amounts of DMSO were added to the sucrose solution of the control group experiments using those compounds.

RNA isolation, reverse-transcription and quantitative PCR for gene expression analysis. RNA was prepared from bees from the described tissues as previously described ${ }^{9}$. Tissues were first manually crushed with a disposable pestle in Trizol Reagent (Invitrogen, San Diego, CA, USA) and RNA was then extracted as per the manufacturer's instructions. RNA was then DNaseI treated by RQ1 RNase-Free DNase (Promega, Madison, WI, USA) and quantified. cDNA was synthesized using approximately $1 \mu \mathrm{g}$ of RNA with the High-Capacity cDNA Reverse Transcription Kit with RNase Inhibitor (Applied Biosystems, Foster City, CA, USA). For quantitative PCR (qPCR) reactions to determine the expression levels of genes of interest, $1 \mu$ of cDNA was used as a template in conjunction with PowerUP SYBR Green Master Mix (Applied Biosystems, Foster City, CA, USA) and appropriate primers. Reactions were run in a LightCycler 480 thermocycler (Basel, Switzerland). Primer sequences targeting transcripts of gene of interest developed for this study are in Supplementary Table 1. Primer sequences for the reference gene $\beta$-actin were from ${ }^{10}$. The difference between the threshold cycle number for $\beta$-actin and that of the gene of interest was used to calculate the level of that gene relative to $\beta$-actin using the typical $2^{(-\Delta \mathrm{CT})}$ method $^{11}$. All qPCR data represents expression values from individual bees (sample sizes found in figure legends) and is displayed as mean \pm SEM. For a given experiment, bees were collected at the same time from a single colony. Each experiment was performed in at least 3 independent trials done on different days. Independent trials sourced bees from different colonies.

RNA-Seq. For RNA-Seq analysis, we used data sets generated previously ${ }^{6-8}$. Briefly, we performed transcriptome profiling (RNASeq) on midguts from bees: (1) fed sucrose solution containing $24 \mu \mathrm{M}$ Tunicamycin or DMSO for $24 \mathrm{~h}^{6}$, (2) maintained at either 35 or $45^{\circ} \mathrm{C}$ for $4 \mathrm{~h}^{7}$, or (3) treated with sucrose solution containing $200 \mu \mathrm{M}$ halofuginone or DMSO for $24 \mathrm{~h}^{8}$. RNASeq analysis was performed on 3 midguts from each group individually. Libraries were prepared using the NEBNext Ultra RNA library preparation kit and then sequenced using the paired end $150 \mathrm{bp}$ sequencing configuration on the Illumina HiSeq 4000 platform. After the sequence reads were trimmed, they were then mapped to the Apis mellifera reference genome then available on NCBI (Amel 4.5 version). After mapping and total gene hit counts calculation, the total gene hit counts table was used for downstream differential expression analysis using DESeq2. The original p-values are generated using the Wald test. The adjusted/corrected p-values are obtained using the Benjamini and Hochberg method. Genes with adjusted $p$-value $<0.05$ and Absolute Log2Fold Change $>1$ were called as significant differentially expressed genes for each comparison. The RNA sequence information in this study was previously submitted to the Gene Expression Omnibus database under the accession numbers GSE139368, GSE159083, and GSE165411 (https:// www.ncbi.nlm.nih.gov/). Venn diagram analysis of gene lists from RNA-Seq data (described previously) was performed using the following webtool (http://bioinformatics.psb.ugent.be/beg/tools/venn-diagrams).

Identification of genes encoding I(2)efl proteins in bee (Hymenoptera: Apoidea: Anthophila) genomes. For a complete list of bee l(2)efl proteins, we used the amino acid sequence of Drosophila mela- 
nogaster 1(2)efl protein (NP_001261156.1) as the query sequence to search select bee genomes in NCBI for which non-redundant protein sequences are available (Apis mellifera, Apis cerana, Megachile rotundata, Eufriesea mexicana, Bombus impatiens, Habropoda laboriosa, Dufourea novaeangliae, Nomia melanderi, Osmia lignaria, Melipona quadrifasciata, Frieseomelitta varia, Megalopta genalis, and Ceratina calcarata). To generate our alignment, we used the amino acid sequences of the Drosophila melanogaster and bee 1(2)efl proteins. The heat shock protein beta-8 of Homo sapiens (NP_055180.1) was used as the outgroup. Protein alignments were generated with MUSCLE $2^{12}$ using default parameters and inspected manually. A maximum-likelihood phylogenetic tree was inferred from the resulting alignment using the RaxML program version $8^{13}$ with the GAMMA model. Bootstrapping was conducted with 100 replicates. The resulting tree was visualized and annotated using ggtree in R. For bee species for which genome annotation was available, we generated graphical representations (not to scale) of the l(2)efl genes in the cluster as well as those outside the cluster (Supplementary Fig. 2). The presence of signal sequences was predicted using Signal 4.1P (http://www.cbs.dtu.dk/services/SignalP/).

RT-LAMP assay. We used RNA extracted as above in conjunction with WarmStart Colorimetric RT-LAMP 2X Master Mix or WarmStart LAMP Kit (DNA \& RNA) (New England Biolabs, Ipswich, MA) and LAMP primers designed using NEB LAMP Primer Design Tool. NEB LAMP Primer Design Tool (Supplementary Table 1). Reactions mixes and conditions were set up according to the manufacturer's instructions. Real-time fluorescence detection of products was performed using an iCycler thermo-cycler (Biorad, Hercules, CA, USA). For real-time fluorescence results, mean \pm SEM is shown and represents relative expression values of $l(2)$ efl 724367 of individual bees calculated using a $2^{(-\Delta \mathrm{CT})}$ method where no reference gene is employed, but instead $\Delta \mathrm{CT}$ is determined by subtracting individual sample values from the mean CT value of the $35^{\circ} \mathrm{C}$ group. For colorimetric results, reactions were stopped at $30 \mathrm{~min}$ and color changes assessed visually. All RT-LAMP data was confirmed in at least 3 independent experiments.

Statistical analysis. Gene expression data was log10 transformed and compared using unpaired t-tests with Welch's correction when values fit normal distributions. We used Mann-Whitney U nonparametric tests when data did not fit normal distributions. We evaluated data normality using Shapiro-Wilk tests. Statistics details can be found in Supplementary Table 2. Survival analysis data was analyzed using Log-rank (Mantel-Cox) test.

\section{Results}

UPR, ISR, and HSR alter the expression of l(2)efl sHSP genes in honey bees. We used Venn diagram analysis to identify a set of differentially expressed genes found in the individual UPR, HSR, and ISR data sets ${ }^{6-8}$. We identified increased and decreased expression of genes involved in multiple processes, but a striking finding was that four genes encoding sHSP (l(2)efl family genes 410087, 724367, 724274, and 724449) were induced in all datasets (Supplementary Table 3). As sHSP genes are involved in stabilizing early unfolded intermediates of proteins and preventing further unfolding and formation of insoluble aggregates (reviewed in Refs. ${ }^{14-17}$ ), these genes appeared to be ideally suited to address diverse proteotoxic stressors.

Bees possess an expanded (I)efl gene family. We previously examined the honey bee genome and identified fourteen genes encoding proteins containing the alpha-crystallin domain characteristic of sHSP (Ref. ${ }^{9}$ and Supplementary Tables 3,4). Ten of these proteins share close homology to sHSP proteins characterized in D. melanogaster. Of these, one is a Heat-shock protein $\beta 1(H s p \beta 1)$ homology while the other nine are most closely related to lethal (2) essential for life $(l(2) e f l)^{18}$. Examination of proteins from select insects representing other orders shows a range in the number of sHSP proteins of the l(2)efl family, from 1 in D. melanogaster to 12 in Folsomia candida (Supplementary Table 5). None of the honey bee l(2)efl proteins are predicted to contain a signal sequence (Supplementary Table 4).

Gene 410087 was originally annotated as being a four-exon gene encoding a protein with two alpha-crystallin domains. PCR-based methods failed to find evidence for this gene model, instead suggesting the existence of two genes, which we will call $410087 a$ and $410087 b$ (both of which are closest to LOC724488 in protein sequence). In addition, gene models from the annotation of genomes from other Apis species (Apis dorsata, Apis florea, and A. cerana) support the model that 410087 is in fact two genes. The proposed mRNA and protein sequences for $410087 a$ and $410087 b$ are included in Supplementary Fig. 1.

Seven of the genes encoding $l(2)$ efl in the honey bee genome are located together in a cluster (Fig. 1A), while the other two are located at other sites in the genome. To explore conservation of this gene cluster in bees and to better understand their relationship to the single $l(2)$ efl genes protein found in D. melanogaster, we used the amino acid sequence of the single $D$. melanogaster $l(2)$ efl to search for $l(2)$ efl encoding genes in select other bee genomes $A$. cerana, $M$. rotundata, E. mexicana, B. impatiens, $H$. laboriosa, D. novaeangliae, $N$. melanderi, $O$. lignaria, M. quadrifasciata, $M$., and C. calcarata). These genomes represent both social and solitary bee species which are broadly distributed phylogenetically within the Clade Anthophila ${ }^{19}$. We found that all bees possessed between 4 and 9 genes encoding $l(2)$ efl sHSP (Supplementary Table 6). For all bee species (except C. calcarata and M. genali) 6-7 clustered $l(2)$ efl genes that were located in a single cluster between thyroid receptor-interacting protein 11 (LOC411348 in A. mellifera) and a gene encoding an uncharacterized protein (LOC100576174 in A. mellifera), while 1-2 (2)efl genes were located elsewhere in the genome.

We used the $l(2)$ efl proteins from above as well as those from F. varia and M. quadrifasciata (bee species for which completely assembled and annotated genomes are not available) to construct a phylogenetic tree. The alpha-crystallin B chain protein of Homo sapiens (NP_001276736.1) was used as the outgroup. (Fig. 1B). 
A Apis meliferra

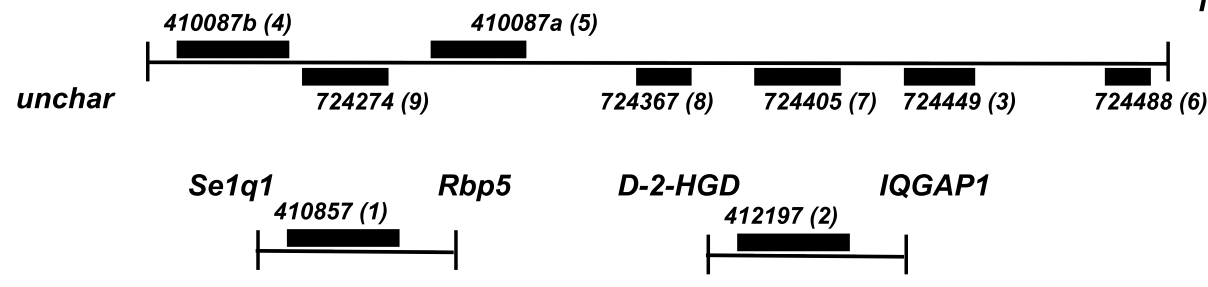

B

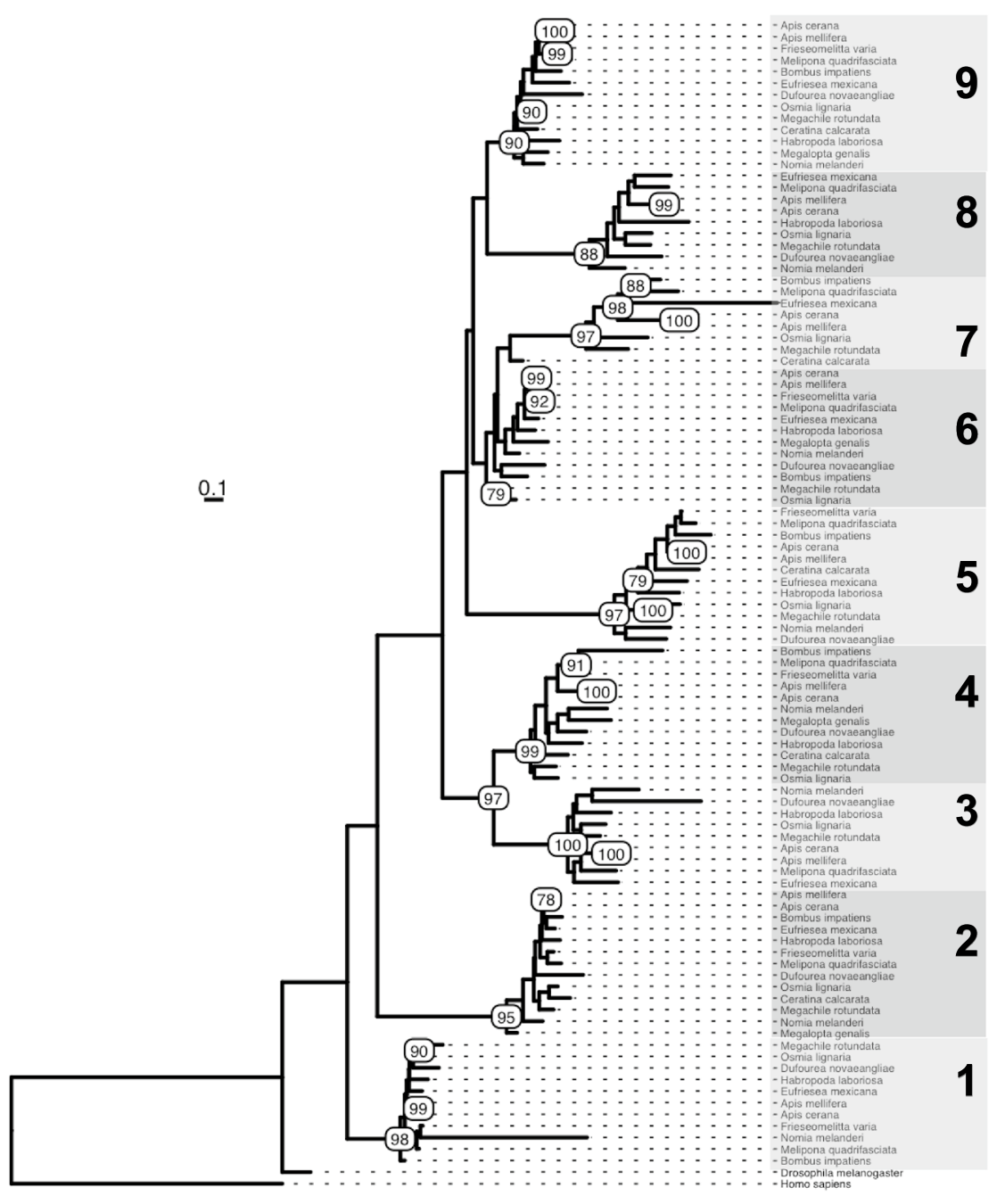

Figure 1. $l(2)$ efl genes and proteins in bees. Schematic of the gene organization of $l(2)$ efl genes in select bee species (both genes found in the cluster as well as genes found outside) (A). Phylogenetic tree of D. melanogaster l(2)efl proteins, bee 1(2)efl proteins (see "Methods"), and H. sapiens alpha-crystallin B protein based on full amino acid sequences. Scale bar represents corresponds to the number of amino acid changes per site (B).

As expected, we found that the bee $l(2) e f l$ proteins are grouped together on the tree and thus appear to share a common relative that expanded in number in bees.

UPR and ISR both induce a subset of $l(2)$ efl sHSP genes in honey bees. Examination of all $l(2) e f l$ genes in three RNAseq datasets showed that in addition to 724367, 410087a, and 724449, the l(2)efl gene 724274 was induced above the cut-off (an adjusted p value of less than 0.05 and a Log2 Fold Change of greater than 1) in the UPR and HSR datasets and was very close to reaching the threshold in ISR dataset and the 724405 gene was induced above the cut-off in the ISR and HSR datasets and was very close to reaching the threshold in UPR 

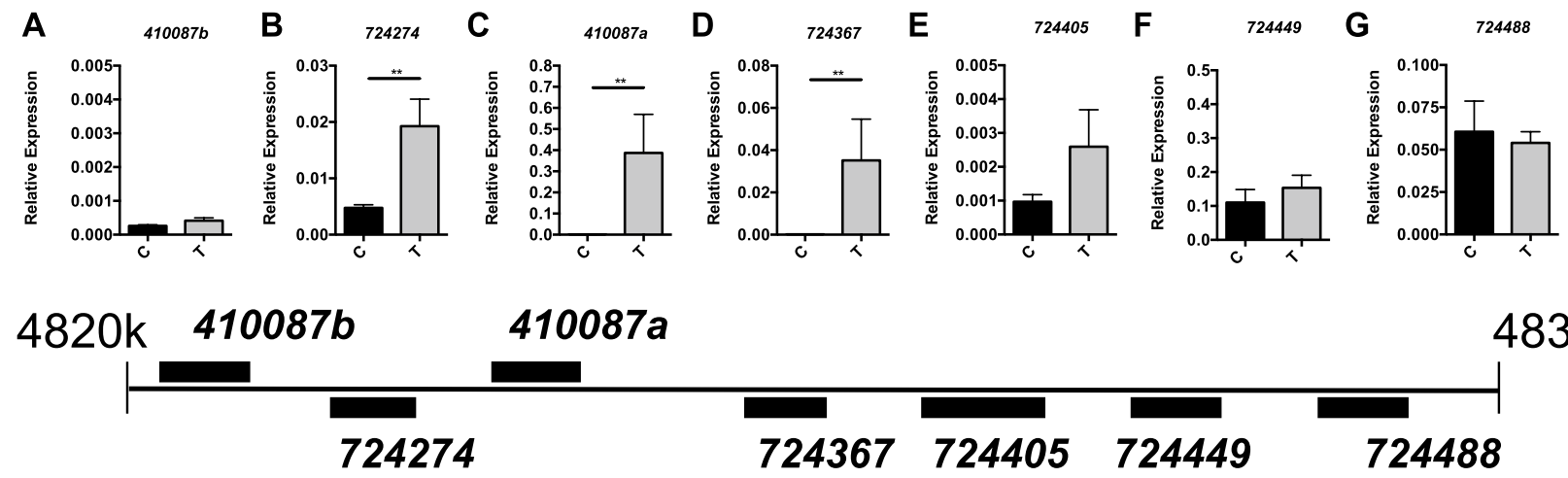

$4839 k$

Figure 2. Select sHSP genes are induced during UPR induction. Transcript levels of the l(2)efl genes $410087 \mathrm{~b}$ (A), 724274 (B), $410087 a(\mathbf{C}), 724367$ (D), 724405 (E), 724449 (F), and 724488 (G) relative to $\beta$-actin in midgut tissue from adult bees captured at the landing board and fed sucrose solution containing tunicamycin $(\mathrm{T}$, $\mathrm{n}=8)$ or vehicle alone $(\mathrm{C}, \mathrm{n}=8)$ for $24 \mathrm{~h}$. A schematic diagram of the colocalized $l(2)$ efl genes in the honey bee genome is shown below for reference. Mean \pm SEM is shown and represents expression values of the genes of interest calculated using the $2^{(-\Delta \mathrm{CT})}$ method for individual bees. Statistical significance is noted as ${ }^{\star} \mathrm{p}<0.05$, and ${ }^{* *} \mathrm{p}<0.01$.
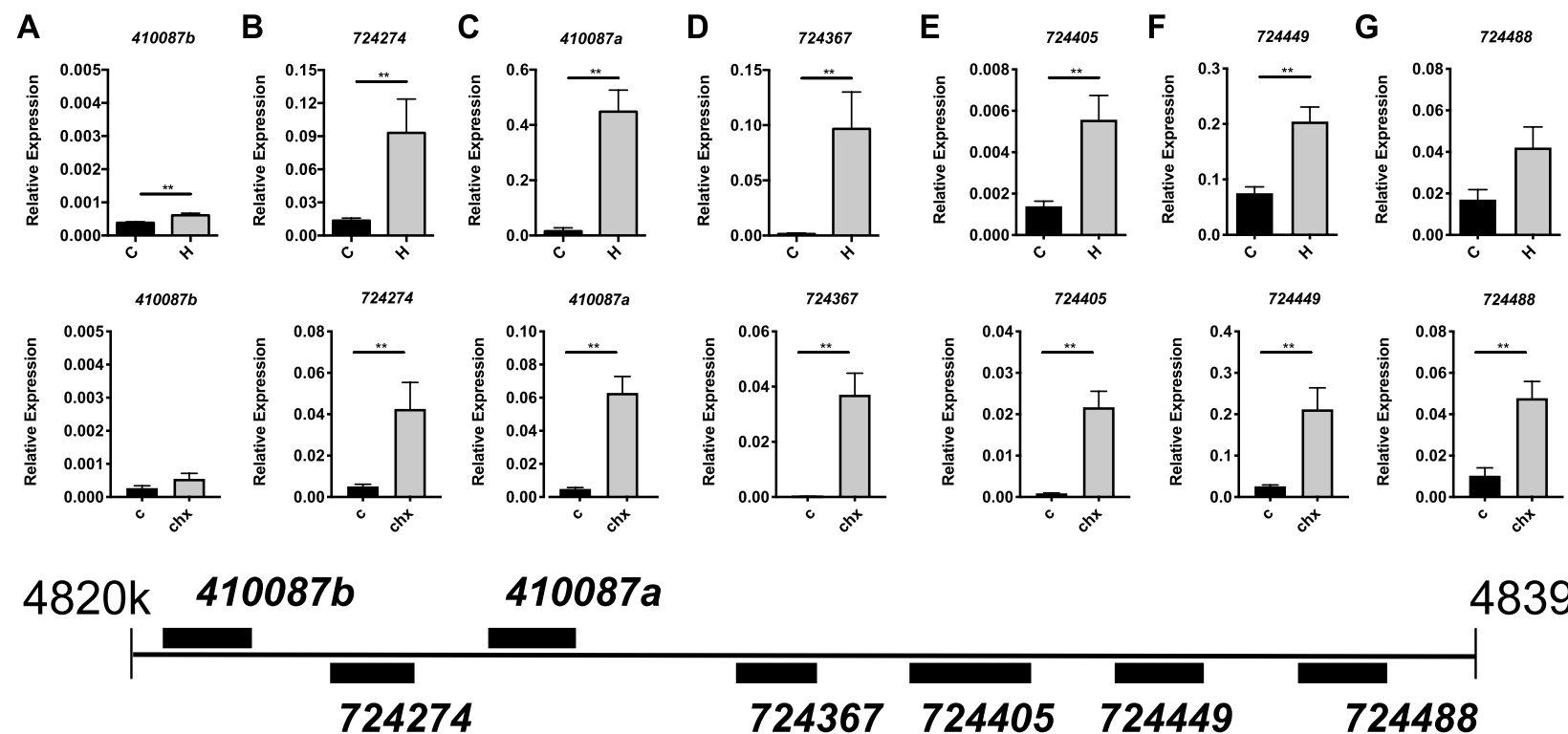

Figure 3. Select sHSP genes are induced during ISR induction. Transcript levels of the $l(2) e f l$ genes $410087 b$ (A), 724274 (B), $410087 a$ (C), 724367 (D), 724405 (E), 724449 (F), and 724488 (G) relative to $\beta$-actin in midgut tissue from adult bees captured at the landing board and fed sucrose solution containing halofuginone $(\mathrm{H}, \mathrm{n}=8)$ or vehicle alone $(\mathrm{C}, \mathrm{n}=8)$ for $24 \mathrm{~h}$ (top row). Transcript levels of these same genes relative to $\beta$-actin in midgut tissue from adult bees captured at the landing board and fed sucrose solution alone $(C, n=8)$ or with $0.5 \mathrm{mg}$ / $\mathrm{ml}$ cyclohexamide for $48 \mathrm{~h}(\mathrm{chx}, \mathrm{n}=8)$ (bottom row). A schematic diagram of the colocalized $l(2) e f l$ genes in the honey bee genome is shown below for reference. Mean \pm SEM is shown and represents expression values of the genes of interest calculated using the $2^{(-\Delta \mathrm{CT})}$ method for individual bees. Statistical significance is noted as ${ }^{*} \mathrm{p}<0.05$, and ${ }^{*} \mathrm{p}<0.01$.

dataset. Thus, 6 out of the 7 l(2)efl genes in the cluster were upregulated by at least 2 of the 3 stressors (even with the low sample number used for the original RNAseq). We then examined the expression of all of the clusterlocalized $l(2)$ efl genes by qPCR. We confirmed that expression of 724274, 724367, 410087a were increased in the midguts of bees treated with tunicamycin relative to control bees (Fig. 2A-G). Statistics details can be found in Supplementary Table 2.

We examined the relative expression of these $l(2)$ efl genes after ISR activation and found that 5 out of 7 were upregulated relative to control bees after halofuginone treatment (Fig. 3A-G, top). $\beta$-actin levels were similar irrespective of tunicamycin or halofuginone treatment as assessed by Ct values (Supplementary Fig. 5). Other work in our lab has suggested that the cellular effects of halofuginone treatment may be due to a disruption in translation as opposed to an amino acid shortage ${ }^{8}$. To investigate whether a direct inhibition of translation leading to RSR activation could also induce l(2)efl sHSP, we used cycloheximide (CHX), which inhibits the translocation 
A

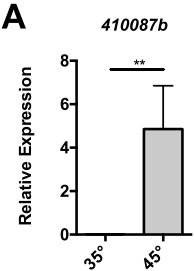

B

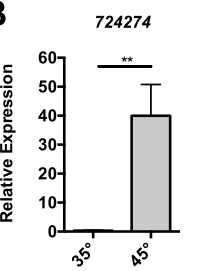

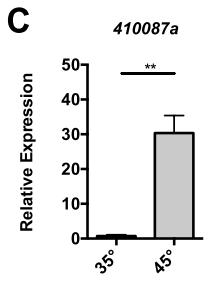
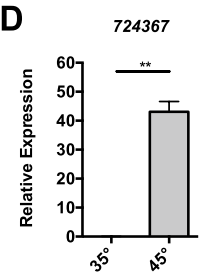

E
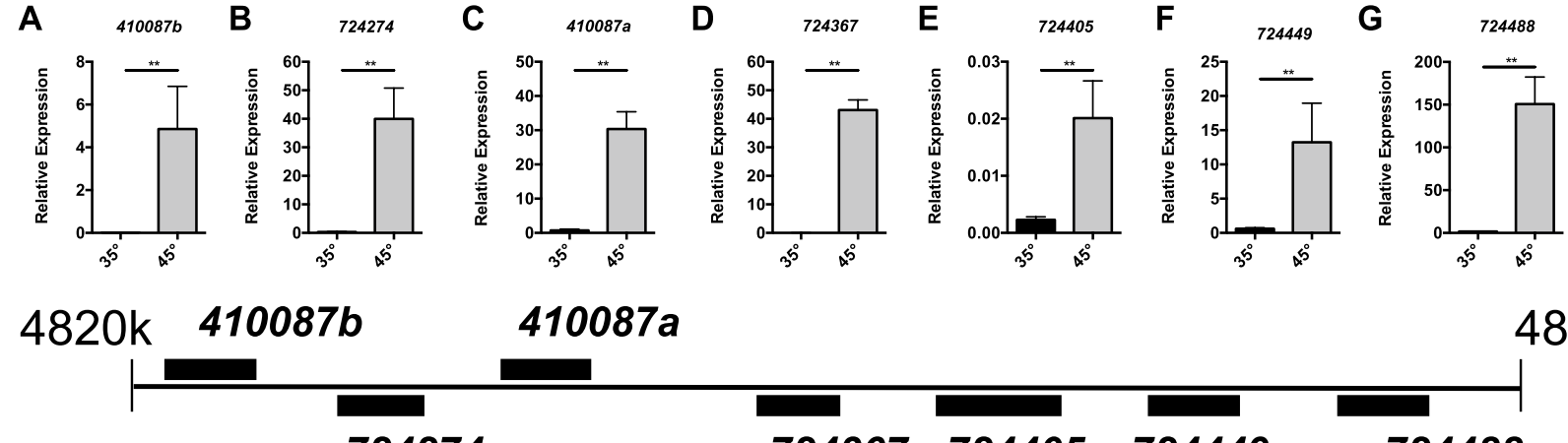

724274

Figure 4. Select sHSP genes are induced during Heat-Shock. Transcript levels of the $l(2)$ efl genes $410087 \mathrm{~b}(\mathbf{A})$, 724274 (B), $410087 a(\mathbf{C}), 724367$ (D), 724405 (E), 724449 (F), and 724488 (G) after heat shock relative to $\beta$-actin in midgut tissue from adult bees captured at the landing board and maintained for $4 \mathrm{~h}$ in cages at either $35^{\circ}$ $(\mathrm{n}=6)$ or $45^{\circ} \mathrm{C}(\mathrm{n}=6)$. A schematic diagram of the colocalized $l(2)$ efl genes in the honey bee genome is shown below for reference. Mean \pm SEM is shown and represents expression values of the genes of interest calculated using the $2^{(-\Delta C T)}$ method for individual bees. Statistical significance is noted as ${ }^{\star} \mathrm{p}<0.05$, and ${ }^{\star *} \mathrm{p}<0.01$.

step in protein synthesis by binding to the ribosome, to block translational elongation. We observed that CHX treatment for $48 \mathrm{~h}$ induces expression of the $l(2)$ efl genes $724488,724405,724367,724274,724449,410087 a$, but not $410087 b$ in the midgut (Fig. 3A-G, bottom). Statistics details can be found in Supplementary Table 2.

HSR induces all l(2)efl sHSP in honey bees. All of the l(2)efl homologs (except 410857) located were induced by heat shock in our dataset (Supplementary Table 1). To confirm these results, we examined heatshock dependent induction of genes encoding proteins that are homologs of existing sHSP (all l(2)efl homologs and $H s p \beta 1)$ in midgut tissue. We observed increased levels of transcripts from the l(2)efl genes 724488,724405 , $724367,724274,724449$, as well as the $410087 a$ and $410087 b$ in the midguts of bees heat shocked at $45^{\circ} \mathrm{C}$ for $4 \mathrm{~h}$ when compare to bees maintained at $35^{\circ} \mathrm{C}$ for that period (Fig. 4). While orally administered treatments appear to reliably only affect the digestive tract, we can apply thermal stress to all tissues equally. Thus, we examined shsp expression in multiple tissues after heat shock. We also observed increased levels of transcripts from the l(2)efl genes $724488,724405,724367,724274,724449,410087 a$ and $410087 b$ after heat shock in other tissues, including the head, thorax, and abdominal wall tissue (Supplementary Fig. 3). Transcripts from the genes $l(2)$ efl (410857) and Heat shock protein beta-1 (Hsp $\beta 1)$ were unchanged after heat shock (Supplementary Fig. 4). $\beta$-actin levels were similar irrespective of temperature as assessed by Ct values (Supplementary Fig. 5). Statistics details can be found in Supplementary Table 2.

Oxidative stress induces a subset of $l(2)$ efl sHSP in honey bees. Oxidative stress is another stressor that can impact proteostasis. We used sodium arsenite (NaArs) and paraquat to model oxidative stress-induced responses in the digestive tract in this species. NaArs is a commonly used elicitor of oxidative stress ${ }^{20-22}$. Arsenite, a common form of arsenic, impacts protein folding directly by reaction with thiols, but is also thought to stimulate ROS production leading to oxidative stress. Paraquat is an herbicide that causes tissue damage to a wide variety of organisms through its generation of the reactive oxygen (ROS) species superoxide after interaction with the mitochondrial electron transport chain $^{23}$. Paraquat has been used to model the sequelae of oxidative stress in multiple species, including C. elegans ${ }^{24}$ and D. melanogaster ${ }^{25}$. To determine appropriate oxidative stress conditions, we performed dose response curves to determine appropriate concentrations of NaArs or paraquat for inducing acute oxidative stress. Based on our results, we chose to use $0.001 \%$ NaArs (Fig. 5A) and $1 \mathrm{mM}$ paraquat (Fig. $5 \mathrm{~B}$ ) as the appropriate dosages to induce acute oxidative stress.

Using qPCR, we examined oxidative stress dependent induction of the seven sHSP genes in midgut tissue after 24 h. For NaArs we observed increased levels of transcripts of all seven after NaArs feeding (Fig. 6A-G, top). Statistics details can be found in Supplementary Table 6. Relative to $\beta$-actin, we observed increased levels of transcripts for $724274,724367,724405,724449$, and 410087 a (but not $410087 b$ or 724488 ) after paraquat treatment (Fig. 6, bottom). $\beta$-actin levels were similar iregardless of NaArs or paraquat or treatment as assessed by Ct values (Supplementary Fig. 5). Statistics details can be found in Supplementary Table 2.

Predicted transcriptional regulation of $l(2)$ efl sHSP genes in honey bees. Based on the diversity of stresses able to promote gene induction, we were interested in exploring the potential trans factors and cis elements responsible for regulating increased gene expression. Focusing on the four $l(2) e f l$ genes we found to be upregulated by all stresses, we sought evidence of conserved transcription factor binding sites (TFBS). In other systems, $s H S P$ genes are regulated by a number of transcription factors, including HSF in both worms $s^{26-28}$ and flies ${ }^{29-31}$. Evidence of HSF-dependent induction of select shsp genes has also been shown in yeast ${ }^{32}$ and mammals ${ }^{33}$. We find evidence of canonical Heat Shock Elements (HSE, consensus sequence=GAANNTTCNNGAA $^{34,35}$ ) at multiple points in these seven genes (Supplementary Fig. 6). We then examined these genes for evidence of direct regulation by UPR-activated transcription factors. Three cis-acting response elements have 
A

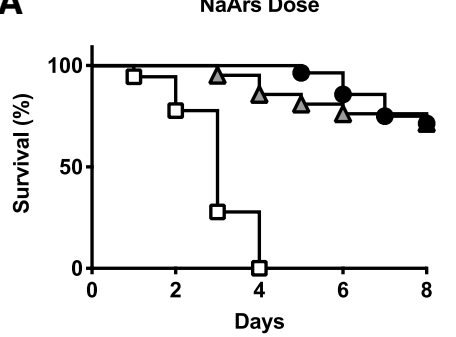

B

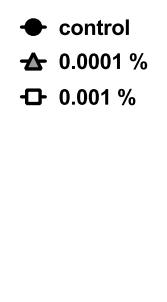

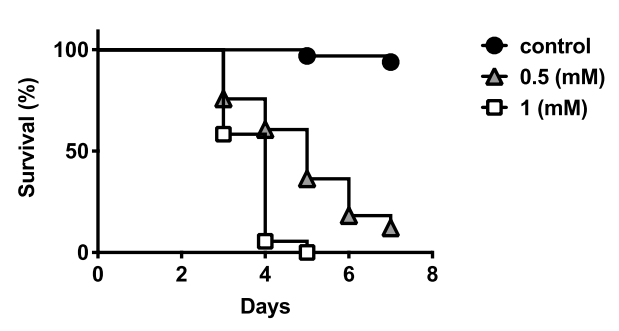

Figure 5. Oxidative stress mediated by NaArs or Paraquat ingestion reduces honey bee survival. (A) Survival of individual honey bees fed sugar solution containing $0(n=28), 0.001 \%(n=18)$ or $0.0001 \%(n=21)$ NaArs. Logrank (Mantel-Cox) test showed that the curves are different (Chi square $=71.83, \mathrm{df}=2,<0.0001)$. (B) Survival of individual honey bees fed sugar solution containing $0(n=33), 1 \mathrm{mM}(\mathrm{n}=36)$, or $0.1 \mathrm{mM}(\mathrm{n}=33)$ paraquat. Log-rank (Mantel-Cox) test showed that the curves are different (Chi square $=90.79, \mathrm{df}=2,<0.0001$ ).
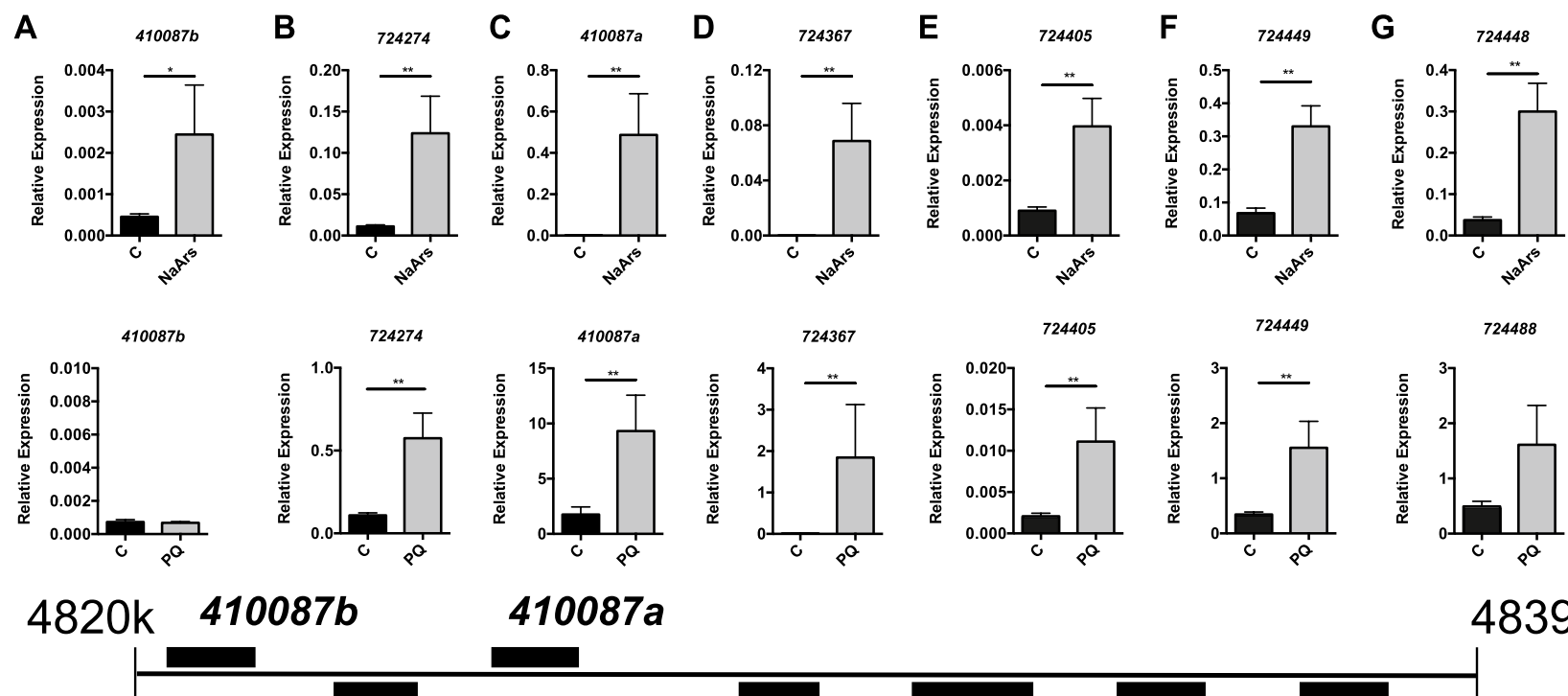

724274

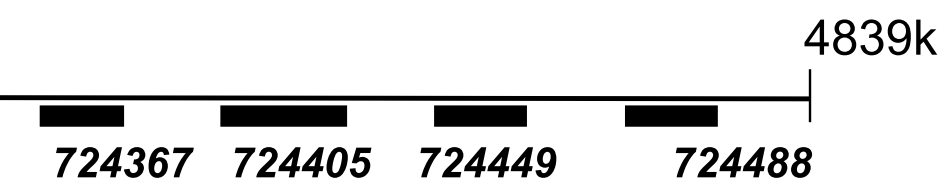

Figure 6. Select sHSP genes are induced during oxidative stress. Transcript levels of the $l(2)$ efl genes $410087 \mathrm{~b}$ (A), $724274(\mathbf{B}), 410087 a(\mathbf{C}), 724367$ (D), $724405(\mathbf{E}), 724449(\mathbf{F})$, and $724488(\mathbf{G})$ relative to $\beta$-actin in midgut tissue from adult bees captured at the landing board and fed sucrose solution alone $(C, n=8)$ or with $0.001 \%$ $\operatorname{NaArs}(n=8)$ (top row) or fed sucrose solution alone $(C, n=8)$ or $1 \mathrm{mM}$ paraquat $(\mathrm{PQ}, \mathrm{n}=8)$ (bottom row) for $24 \mathrm{~h}$. A schematic diagram of the colocalized $l(2)$ efl genes in the honey bee genome is shown below for reference. Mean \pm SEM is shown and represents expression values of the genes of interest calculated using the $2^{(-\Delta C T)}$ method for individual bees. Statistical significance is noted as ${ }^{\star} \mathrm{p}<0.05$, and ${ }^{\star *} \mathrm{p}<0.01$.

been characterized in the promoter regions of UPR-induced genes in mammals. These are the ERSE (ER Stress Response Element, CCAATN9CCACG), the ERSE-II (ER Stress Response Element II, ATTGGNCCACG), and the UPRE (Unfolded Protein Response Element, TGACGTGR) (Reviewed in Ref. ${ }^{36}$ ). While these sites were not evident, there were a number of instances of another ATF4-binding motif, TTKCATCAK that has recently been characterized in mammals ${ }^{37}$ and shown to regulate gene expression in insects ${ }^{38}$ (Supplementary Fig. 6). The transcriptional regulator controlling the oxidative stress response, NRF2 (and its homologs), are responsible for promoting resistance to oxidative stress in C. elegans ${ }^{39}, D$. melanogaster ${ }^{21}$, and mammals (reviewed in Ref. ${ }^{40}$ ). The Nrf2 signaling system is functionally and structurally conserved in D. melanogaster ${ }^{41}$, which possess both a Nrf2 homolog, the Cap'n'collar splice form $\mathrm{C}(\mathrm{CncC})$ protein, and the E3 ubiquitin ligase that regulates it, Kelch-like ECH-associated protein 1 (Keap1). Honey bee possess genes encoding CncC (LOC725081) and Keap1 (LOC411679). Nrf2 responsive genes possess the consensus binding site (TGAYNNNGC) which makes up the core of the antioxidant responsive element (ARE) ${ }^{21}$. We found a number of these TGAYNNNGC sites near these four genes (Supplementary Fig. 6). In addition to NRF2, the transcription factor FOXO has been shown to be an important regulator of oxidative stress responses and can upregulate transcription of sHSP genes in both worms ${ }^{26-28}$ and flies ${ }^{29-31}$. We observed a number of consensus sites for FOXO binding (TKTTYACY ${ }^{42}$ ) at multiple points in these four genes (Supplementary Fig. 6). 
A

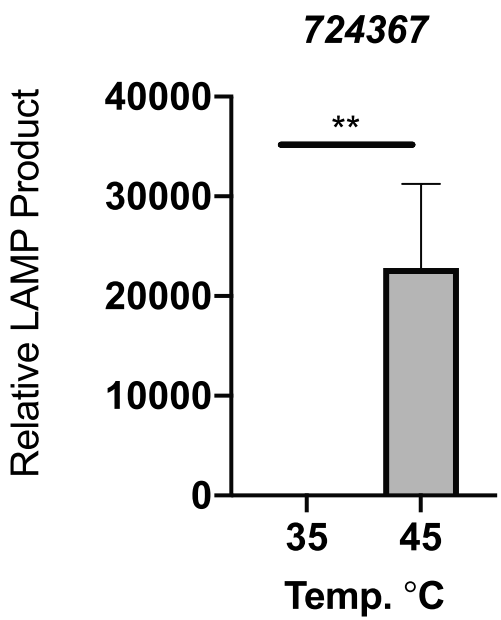

B

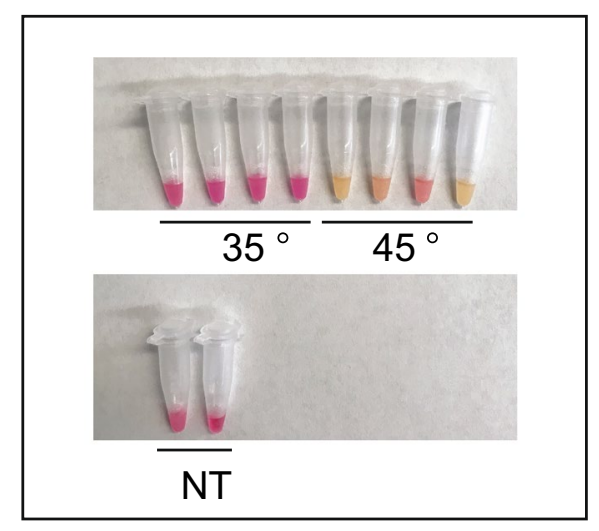

Figure 7. RT-LAMP assay detects heat-induced induction of the $724367 l(2)$ efl gene. Transcript levels of the $l(2)$ efl 724367 gene in midgut tissue from adult bees captured at the landing board and maintained for $4 \mathrm{~h}$ in cages at either $35^{\circ} \mathrm{C}$ or $45^{\circ} \mathrm{C}$ as assessed by RT-LAMP using quantitative real-time fluorescence (A) or end-point colorimetric (B) read-outs. For real-time fluorescence, mean \pm SEM is shown and represents relative expression values of $l(2)$ efl 724367 (see "Methods" for details). Statistical significance is noted as ${ }^{\star} \mathrm{p}<0.05$, and ${ }^{\star *} \mathrm{p}<0.01$.

LAMP assay detection of $l(2) e f l$ sHSP genes. We developed an RT-LAMP assay to measure the relative amount of transcript from the 724367 l(2)efl gene. We chose this gene as it is induced by the most stressors and demonstrates the highest level of induction. We observed that our RT-LAMP assay can detect increased 724367 expression in the midguts of bees heat shocked at $45^{\circ} \mathrm{C}$ for $4 \mathrm{~h}$ when compared to bees maintained at $35^{\circ} \mathrm{C}$ for that period using real-time fluorescence relative quantification (Fig. $7 \mathrm{~A}, \mathrm{p}=0.0002, \mathrm{t}=8.742, \mathrm{df}=5.456$ ). We also found that we could easily differentiate samples from control or heat-shocked bees using visual assessment of an end-point colorimetric read out (Fig. 7B).

\section{Discussion}

As no single cause for the recent increase in honey bee disease has been found, there is intensified focus on the synergistic impact of disparate stressors working in concert. A critical first step in understanding such combinatorial impacts involves defining specific common cellular processes that are impacted by multiple stressors and could consequently serve as links to cellular dysfunction, tissue pathology, disease, and mortality in honey bees ${ }^{43}$. The various pathways that make up cellular responses to disruptions in proteostasis represent one set of processes to examine for such interactions. We used an unbiased approach to identify novel genes altered in expression by induction of three proteostatic network responses in the honey bee using transcriptome profiling (RNASeq) ${ }^{6-8}$. We hypothesized that we could then utilize this information to better understand how these stresses interact to impact honey bee health and to identify candidate biomarkers of stress in honey bees. Here we report that genes encoding sHSP, sometimes known as the 'first line of defense' against protein folding stress, (reviewed in Refs. ${ }^{14-17}$ ), are part of a common cellular response to a broad array of stressors. In other invertebrates, such as $D$. melanogaster and C. elegans, sHSP are involved in maintaining proteostasis in the face of diverse environmental insults. D. melanogaster possess $12 \mathrm{sHSP}$-coding genes ${ }^{44}$. Ten of these have been shown to be upregulated by heat stress, while seven are affected by oxidative stress. Notably, the single D. melanogaster $(l(2)$ efl $)$ has been shown to be part of a core stress response to diverse stresses along with HSP70 and HSP83 ${ }^{45,46}$. C. elegans, contains 16 genes encoding sHSP. As with those in D. melanogaster, some genes are inducible by stress, while others are not (reviewed in Ref. ${ }^{47}$ ). Outside of the highly conserved $\alpha$-crystallin domain, these proteins display significant areas of low homology in the N-terminal and C-terminal regions and range in size from 12 to $42 \mathrm{kD}$.

With some exceptions, our phylogenetic analysis of the sHSP proteins in other bee species shows a high degree of conservation of this family among bees from different life histories. Examination of the underlying gene structure also shows striking conservation of the shsp gene cluster. However, shsp number and gene structure do not obviously correspond to the known phylogenetic relationships among bees ${ }^{19}$. For example, M. genalis and C. calcarata, which are both found along with A. mellifera in the Apidae family, have reduced numbers of shsp genes and a remarkably divergent gene structure while $M$. rotundata and O. lignaria, which are both in the Megachilidae family, share shsp gene number and structure with A. mellifera. Due to their critical role in pollination in natural ecosystems, there is great interest in understanding the factors influencing the health of non-Apis bee species ${ }^{48}$. Thus, future work to better understand the evolutionary context for these differences and to define whether various bee species have different stress responses and tolerances will be important. In addition, while not currently, validated, our results suggest that shsp may provide potential biomarkers of stress in some non-Apis bee species.

In other model systems, studies of diverse stressors and their cognate response pathways has uncovered both unique and common gene targets. In D. melanogaster for example, heat shock, oxidative stress, and ionizing 
radiation all upregulated a core set of shared genes, although unique gene targets were associated with each stress trigger ${ }^{45}$. Multiple studies have also shown that interactions between stress pathways are often complex. For example, the OSR is inhibited by heat stress in C. elegans ${ }^{49}$ while it synergizes with the UPR ${ }^{50}$. In D. melanogaster, l(2)efl has been shown to be part of a core stress response to diverse stresses along with HSP70 and HSP $83^{45,46}$. In other organisms, $s H S P$ genes have been shown to be regulated by a number of transcription factors, including HSF (in both worms ${ }^{26-28}$ and flies ${ }^{29-31}$ ) and FOXO (in both worms ${ }^{26-28}$ and flies ${ }^{29-31}$ ). Our TFBS analysis suggests that the honey bee $s H S P$ genes located in the cluster are potentially regulated by HSF, ATF4, NRF2, and FOXO, likely acting in concert. However, future studies will be required to determine the functional importance of these TFBS as the sequence motifs responsible for the binding of most transcription factors have not been empirically tested in the honey bee.

Our work is the first report of sHSP genes being transcriptionally upregulated by the UPR. The cellular benefit of increasing cytoplasmic sHSP protein production in the face of UPR (and ISR) is not clear. For the UPR, triggered by unfolded protein build-up in the ER, it is possible that increasing sHSP expression in the cytoplasm is important to help respond to the increased transport of these unfolded proteins to the cytoplasm for degradation by the ERAD pathway. For the ISR, activated by halofuginone or cyclohexamide, translational pausing might be expected to reduce the typical burden of unfolded proteins in the cytoplasm. However, the ISR and UPR all result in reduced protein synthesis. This impact of inhibition on different proteins is uneven and may contribute to stoichiometric differences that disrupt the assembly of protein complexes resulting in the need to protect against aggregate formation. Such issues have been observed during mito-nuclear protein imbalances caused by disruption of mitochondrial protein synthesis ${ }^{51}$.

As declining health in a honey bee colony is often caused by interacting stresses, being able to quantify individual stressors such as infectious agents or pesticides in and around colonies is critical for monitoring possible exposures to disease-causing agents. However, for identifying resultant unhealthy colonies, the ability to measure the relative stress of individual bees or the colony as a whole through the use of biomarkers represents a key diagnostic tool ${ }^{48}$. Quantification of cellular stress responses, often through measuring of HSP, has been used as a surrogate to identify general organismal stress in a variety of settings, including the honey bee (reviewed in Ref. ${ }^{3}$ ). Based on the robust induction of some of the $l(2)$ efl genes in response to a broad array of stressors, quantification of these sHSP may provide an optimal biomarker for honey bee stress. Recently, another group proposed sHSP of the $l(2)$ efl family as potential biomarkers for honey bee queen health after they were identified using a proteomics discovery strategy ${ }^{52,53}$. In their study, these authors found that the proteins encoded by the l(2)efl genes 724367 and 412197 had some limitations for predicting stressed bees. This group also discussed a number of key aspects of diagnostic testing that will be critical to explore in future work, including the doseresponsiveness of the potential biomarker and the time-course of induction after exposure. Other aspects to consider include factors that may impact stress responses such as age or caste. Some of the sHSP genes discussed here have been shown to be part of the antiviral response in honey bees and bumble bees ${ }^{54,55}$. Finally, as a new biomarker used to measure individual bee stress, the levels of $l(2)$ efl gene expression have not yet been linked to colony-level outcomes. As an example, the expression of yolk protein Vitellogenin and select immune genes (as well as abdominal lipid levels) were used to link individual bee health to colony health and survival in a seminal biomarker study ${ }^{56}$.

For biomarker detection, a point-of-care testing (POCT) assay would be the most useful. Future progress in a number of key areas would be necessary for making any new POCT strategies both desirable and feasible for use by beekeepers in the field. First, sample preparation methods that are simple, inexpensive, and provide an adequate sample size need to be established. Second, a rugged assay that consistently provides reproducible and accurate results is critical. Third, robust and easy to use hardware and software that maximize user benefits and promote adoption must be developed. Finally, any new assays need to be compared to the current gold-standard diagnostic tools and coupled with empirically derived management recommendations. LAMP assays have many features that make it ideal for POCT analysis, including simplified sample preparation potential and fast reaction times using inexpensive equipment (reviewed in Ref. ${ }^{57}$ ). However, the hardware and software used to run and analyze assays still represent limiting factors. In recent years there has been burgeoning interest in coupling diverse POCT strategies with the smartphone as a measurement and analysis platform ${ }^{58}$ due to its ubiquity and versatility ${ }^{59}$. Attempts to couple LAMP with detection using smartphone optics have been quite successful in other settings $^{60-62}$, allowing for inexpensive quantification and analysis of results. Since the advent of COVID-19, there has been explosive progress in both the assays ${ }^{63}$ and the specialized accessories ${ }^{64}$ required for such assays, making this the ideal time to pursue LAMP-based strategies for diagnosing honey bee disease more broadly. The ability of recent developments in 3D printing technology to facilitate advances in POCT are particularly exciting ${ }^{65}$. For example, Nguyen et al. designed and fabricated a single accessory that can perform the heating required for the LAMP reaction and assist in the imaging using a smartphone ${ }^{66}$.

Transcriptome profiling identified a subset of sHSP of the lethal (2) essential for life (l(2)efl) gene family as common transcriptional targets activated by diverse cellular stressors. Interestingly, this work is the first report of sHSP genes being transcriptionally upregulated by the UPR and ISR. Our data suggests that $l(2)$ efl genes are part of a core stress response that might provide an effective biomarker for cellular stress in honey bees. We designed and tested an RT-LAMP assay to detect increased $l(2)$ efl gene expression in response to heat-stress, thus providing a powerful proof of principle for using $l(2)$ efl gene expression as a biomarker of stress. Future work will be necessary to link changes in $s H S P$ gene expression to colony-level outcomes, to adapt our preliminary assay into Point of Care Testing (POCT) assay appropriate for use as a diagnostic tool for use in the field, and to couple assay results to management recommendations. 
Received: 27 March 2021; Accepted: 29 October 2021

Published online: 11 November 2021

\section{References}

1. Potts, S. G. et al. Global pollinator declines: Trends, impacts and drivers. Trends Ecol. Evol. (Amst.) 25, 345-353 (2010).

2. Steinhauer, N. et al. Drivers of colony losses. Curr. Opin. Insect Sci. 26, 142-148 (2018).

3. Even, N., Devaud, J.-M. \& Barron, A. General stress responses in the honey bee. Insects 3, 1271-1298 (2012).

4. Taylor, R. C., Berendzen, K. M. \& Dillin, A. Systemic stress signalling: Understanding the cell non-autonomous control of proteostasis. Nat. Rev. Mol. Cell Biol. 15, 211-217 (2014).

5. Walter, P. \& Ron, D. The unfolded protein response: From stress pathway to homeostatic regulation. Science 334, 1081-1086 (2011).

6. Adames, T. R. et al. The IRE1 pathway regulates honey bee Unfolded Protein Response gene expression. Insect Biochem. Mol. 121, 103368. https://doi.org/10.1016/j.ibmb.2020.103368 (2020).

7. Bach, D. M. et al. Thermal stress induces tissue damage and a broad shift in regenerative signaling pathways in the honey bee digestive tract. J. Exp. Biol. 224, jeb242262 (2021).

8. Flores, M. E., McNamara-Bordewick, N. K., Lovinger, N. L. \& Snow, J. W. Halofuginone triggers a transcriptional program centered on ribosome biogenesis and function in honey bees. Insect Biochem. Mol. 139, 103667 (2021).

9. McKinstry, M., Chung, C., Truong, H., Johnston, B. A. \& Snow, J. W. The heat shock response and humoral immune response are mutually antagonistic in honey bees. Sci. Rep. 7, 8850 (2017).

10. van Englesdorp, D. et al. Colony collapse disorder: A descriptive study. PLoS One 4, e6481 (2009).

11. Schmittgen, T. D. \& Livak, K. J. Analyzing real-time PCR data by the comparative C(T) method. Nat. Protoc. 3, 1101-1108 (2008).

12. Edgar, R. C. MUSCLE: Multiple sequence alignment with high accuracy and high throughput. Nucleic Acids Res. 32, 1792-1797 (2004).

13. Stamatakis, A. RAxML version 8: A tool for phylogenetic analysis and post-analysis of large phylogenies. Bioinformatics 30, 1312-1313 (2014).

14. Basha, E., O’Neill, H. \& Vierling, E. Small heat shock proteins and. Trends Biochem. Sci. 37, 106-117 (2012).

15. Haslbeck, M. \& Vierling, E. A first line of stress defense: Small heat shock proteins and their function in protein homeostasis. J. Mol. Biol. 427, 1537-1548 (2015).

16. Strauch, A. \& Haslbeck, M. The function of small heat-shock proteins and their implication in proteostasis. Essays Biochem. 60, 163-172 (2016).

17. Bakthisaran, R., Tangirala, R. \& Rao, C. M. Small heat shock proteins: Role in cellular functions and pathology. Biochim. Biophys. Acta BBA Proteins Proteom. 1854, 291-319 (2015).

18. Kurzik-Dumke, U. \& Lohmann, E. Sequence of the new Drosophila melanogaster small heat-shock-related gene, lethal(2) essential for life [1(2) efl], at locus 59F4,5. Gene 154, 171-175 (1995).

19. Hedtke, S. M., Patiny, S. \& Danforth, B. N. The bee tree of life: A supermatrix approach to apoid phylogeny and biogeography. BMC Evol. Biol. 13, 138-213 (2013).

20. Hughes, M. F. Arsenic toxicity and potential mechanisms of action. Toxicol. Lett. 133, 1-16 (2002).

21. Sykiotis, G. P. \& Bohmann, D. Keap1/Nrf2 signaling regulates oxidative stress tolerance and lifespan in Drosophila. Dev. Cell 14, 76-85 (2008).

22. Sahu, S. N. et al. Genomic analysis of stress response against arsenic in Caenorhabditis elegans. PLoS One 8, e66431 (2013).

23. Cocheme, H. M. \& Murphy, M. P. Complex I is the major site of mitochondrial superoxide production by paraquat. J. Biol. Chem. 283, 1786-1798 (2008).

24 Keith, S. A., Amrit, F. R. G., Ratnappan, R. \& Ghazi, A. The C. elegans healthspan and stress-resistance assay toolkit. Methods 68, 476-486 (2014).

25. Girardot, F., Monnier, V. \& Tricoire, H. Genome wide analysis of common and specific stress responses in adult drosophila melanogaster. BMC Genom. 5, 74 (2004).

26. Hsu, A. L., Murphy, C. T. \& Kenyon, C. Regulation of aging and age-related disease by DAF-16 and heat-shock factor. Science 300, $1142-1145$ (2003).

27. Murphy, C. T. et al. Genes that act downstream of DAF-16 to influence the lifespan of Caenorhabditis elegans. Nature 424, 277-284 (2003).

28. Morley, J. F. \& Morimoto, R. I. Regulation of longevity in Caenorhabditis elegans by heat shock factor and molecular chaperones. Mol. Biol. Cell 15, 657-664 (2004).

29. Klemenz, R. \& Gehring, W. J. Sequence requirement for expression of the Drosophila melanogaster heat shock protein hsp22 gene during heat shock and normal development. Mol. Cell Biol. 6, 2011-2019 (1986).

30. Donovan, M. R. \& Marr, M. T. II. dFOXO activates large and small heat shock protein genes in response to oxidative stress to maintain proteostasis in Drosophila. J. Biol. Chem. 291, 19042-19050 (2016).

31. Wang, M. C., Bohmann, D. \& Jasper, H. JNK extends life span and limits growth by antagonizing cellular and organism-wide responses to insulin signaling. Cell 121, 115-125 (2005).

32. Solís, E. J. et al. Defining the essential function of yeast Hsf1 reveals a compact transcriptional program for maintaining eukaryotic proteostasis. Mol. Cell 63, 60-71 (2016).

33. Mahat, D. B., Salamanca, H. H., Duarte, F. M., Danko, C. G. \& Lis, J. T. Mammalian heat shock response and mechanisms underlying its genome-wide transcriptional regulation. Mol. Cell 62, 63-78 (2016).

34. Birch-Machin, I. et al. Genomic analysis of heat-shock factor targets in Drosophila. Genome Biol. 6, R63 (2005).

35. Guertin, M. J. \& Lis, J. T. Chromatin landscape dictates HSF binding to target DNA elements. PLoS Genet. 6, e1001114 (2010).

36. Samali, A., FitzGerald, U., Deegan, S. \& Gupta, S. Methods for monitoring endoplasmic reticulum stress and the unfolded protein response. Int. J. Cell Biol. 2010, 1-11 (2010)

37. Han, J. et al. ER-stress-induced transcriptional regulation increases protein synthesis leading to cell death. Nat. Cell Biol. 15, 481-490 (2013).

38. Lee, J. E., Oney, M., Frizzell, K., Phadnis, N. \& Hollien, J. Drosophila melanogaster activating transcription factor 4 regulates glycolysis during endoplasmic reticulum stress. G3 (Bethesda) 5, 667-675 (2015).

39. An, J. H. \& Blackwell, T. K. SKN-1 links C. elegans mesendodermal specification to a conserved oxidative stress response. Genes Dev. 17, 1882-1893 (2003).

40 Sykiotis, G. P. \& Bohmann, D. Stress-activated cap'n'collar transcription factors in aging and human disease. Sci. Signal 3, re3 (2010).

41. Pitoniak, A. \& Bohmann, D. Free radical biology and medicine. Free Radic. Biol. Med. 88, 302-313 (2015).

42. Bai, H., Kang, P., Hernandez, A. M. \& Tatar, M. Activin signaling targeted by insulin/dFOXO regulates aging and muscle proteostasis in Drosophila. PLoS Genet. 9, e1003941-e1004016 (2013).

43. Johnston, B. A., Hooks, K. B., McKinstry, M. \& Snow, J. W. Divergent forms of endoplasmic reticulum stress trigger a robust unfolded protein response in honey bees. J. Insect Physiol. 86, 1-10 (2016). 
44. Morrow G., Tanguay R.M. (2015) Drosophila Small Heat Shock Proteins: An Update on Their Features and Functions. In: Tanguay R., Hightower L. (eds) The Big Book on Small Heat Shock Proteins. Heat Shock Proteins, vol 8. Springer, Cham. https://doi.org/ 10.1007/978-3-319-16077-1_25 (2015).

45. Landis, G., Shen, J. \& Tower, J. Gene expression changes in response to aging compared to heat stress, oxidative stress and ionizing radiation in Drosophila melanogaster. Aging (Albany NY) 4, 768-789 (2012).

46 Shen, J. \& Tower, J. Aging, MnSOD, and hormesis mechanisms converge on liver mUPR. Cell Cycle 12, 1 (2013).

47. Candido, E. P. M. The small heat shock proteins of the nematode Caenorhabditis elegans: Structure, regulation and biology. Prog. Mol. Subcell. Biol. 28, 61-78 (2002).

48. López-Uribe, M. M., Ricigliano, V. A. \& Simone-Finstrom, M. Defining pollinator health: Assessing bee ecological, genetic, and physiological factors at the individual, colony, and population levels. Annual Review of Animal Biosciences. 8, 269-294. (2020).

49. Crombie, T. A., Tang, L., Choe, K. P. \& Julian, D. Inhibition of the oxidative stress response by heat stress in Caenorhabditis elegans. J. Exp. Biol. 219, 2201-2211 (2016).

50. Glover-Cutter, K. M., Lin, S. \& Blackwell, T. K. Integration of the unfolded protein and oxidative stress responses through SKN-1/ Nrf. PLoS Genet. 9, e1003701 (2013).

51. Houtkooper, R. H. et al. Mitonuclear protein imbalance as a conserved longevity mechanism. Nature 497, 451-457 (2014).

52. McAfee, A. et al. Vulnerability of honey bee queens to heat-induced loss of fertility. Nat. Sustain. 3, 367-376 (2020).

53 McAfee, A. et al. Candidate stress biomarkers for queen failure diagnostics. BMC Genom. 21, 1-12. https://doi.org/10.1186/ s12864-020-06992-2 (2020).

54 Brutscher, L. M., Daughenbaugh, K. F. \& Flenniken, M. L. Virus and dsRNA-triggered transcriptional responses reveal key components of honey bee antiviral defense. Sci. Rep. https://doi.org/10.1038/s41598-017-06623-z (2017).

55. McMenamin, A., Daughenbaugh, K., Parekh, F., Pizzorno, M. \& Flenniken, M. Honey bee and bumble bee antiviral defense. Viruses 10, 395-422 (2018).

56. Smart, M., Pettis, J., Rice, N., Browning, Z. \& Spivak, M. Linking measures of colony and individual honey bee health to survival among apiaries exposed to varying agricultural land use. PLoS One 11, e0152685 (2016).

57. Notomi, T., Mori, Y., Tomita, N. \& Kanda, H. Loop-mediated isothermal amplification (LAMP): Principle, features, and future prospects. J. Microbiol. 53, 1-5 (2015).

58. Vashist, S. K., Luppa, P. B., Yeo, L. Y., Ozcan, A. \& Luong, J. H. T. Emerging technologies for next-generation point-of-care testing. Trends Biotechnol. 33, 692-705 (2015).

59. Ozcan, A. Mobile phones democratize and cultivate next-generation imaging, diagnostics and measurement tools. Lab Chip 14, 3187-3194 (2014).

60. Stedtfeld, R. D. et al. Gene-Z: A device for point of care genetic testing using a smartphone. Lab Chip 12, 1454-1459 (2012).

61 Priye, A. et al. A smartphone-based diagnostic platform for rapid detection of Zika, chikungunya, and dengue viruses. Sci. Rep. 7, 44778. https://doi.org/10.1038/srep44778 (2017).

62. Kong, J. E. et al. Highly stable and sensitive nucleic acid amplification and cell-phone-based readout. ACS Nano 11, 2934-2943 (2017).

63. Esbin, M. N. et al. Overcoming the bottleneck to widespread testing: A rapid review of nucleic acid testing approaches for COVID19 detection. RNA 26, 771-783 (2020).

64. Ding, X., Mauk, M. G., Yin, K., Kadimisetty, K. \& Liu, C. Interfacing pathogen detection with smartphones for point-of-care applications. Anal. Chem. 91, 655-672 (2019).

65 Chan, H. N., Tan, M. J. A. \& Wu, H. Point-of-care testing: Applications of 3D printing. Lab Chip 17, 2713-27397. https://doi.org/ 10.1039/C7LC00397H (2017).

66. Nguyen, H. V., Nguyen, V. D., Liu, F. \& Seo, T. S. An integrated smartphone-based genetic analyzer for qualitative and quantitative pathogen detection. ACS Omega 5, 22208-22214 (2020).

\section{Acknowledgements}

The authors thank Melissa Flores and Lena Kogan for helpful comments and critical review of the manuscript.

\section{Author contributions}

S.R.S. and J.W.S. conceived and designed the experiments. S.R.S., D.B., N.C.R., J.S., N.L.L., A.J.L., and J.W.S. performed honey bee experiments and analyzed the data. All authors contributed to the drafting and revision of the article.

\section{Funding}

J.W.S. thanks Project Apis m. for their generous support in completion of this project.

\section{Competing interests}

The authors declare no competing interests.

\section{Additional information}

Supplementary Information The online version contains supplementary material available at https://doi.org/ 10.1038/s41598-021-01547-1.

Correspondence and requests for materials should be addressed to J.W.S.

Reprints and permissions information is available at www.nature.com/reprints.

Publisher's note Springer Nature remains neutral with regard to jurisdictional claims in published maps and institutional affiliations. 
(c) (i) Open Access This article is licensed under a Creative Commons Attribution 4.0 International cc) License, which permits use, sharing, adaptation, distribution and reproduction in any medium or format, as long as you give appropriate credit to the original author(s) and the source, provide a link to the Creative Commons licence, and indicate if changes were made. The images or other third party material in this article are included in the article's Creative Commons licence, unless indicated otherwise in a credit line to the material. If material is not included in the article's Creative Commons licence and your intended use is not permitted by statutory regulation or exceeds the permitted use, you will need to obtain permission directly from the copyright holder. To view a copy of this licence, visit http://creativecommons.org/licenses/by/4.0/.

(C) The Author(s) 2021 